\title{
Electron Spins in Self-Assembled (In,Ga)As/GaAs Quantum Dots Studied by Pump-Probe Faraday Rotation
}

\author{
A. Greilich, I.A. Yugova, R. Oulton, E.A. Zhukov, \\ D.R. YAKovleV, M. Bayer
}

Experimentelle Physik II, Universität Dortmund, 44221 Dortmund, Germany

\author{
A. Shabaev, Al.L. Efros \\ Naval Research Laboratory, Washington, DC 20375, USA \\ V. Stavarache, D. Reuter and A.D. Wieck \\ Angewandte Festkörperphysik, Ruhr-Universität Bochum \\ 44780 Bochum, Germany \\ Static and dynamic properties of electron spins in self-assembled \\ (In, Ga)As/GaAs quantum dots which contain on average a single electron \\ per dot were studied by pump-probe Faraday rotation. Examples given here \\ are the $g$-factor tensor components as well as the dephasing time $T_{2}^{*}$ within
} a dot ensemble.

PACS numbers: 78.55.Cr, 78.67.Hc

\section{Introduction}

The spin of an electron isolated in a quantum dot (QD) has attracted considerable interest for solid state quantum information processing $[1,2]$. This interest is based on that many of the mechanisms which lead to spin relaxation are suppressed in QDs with their discretized energy level spectrum. The only relevant mechanisms for electrons are the spin-orbit interaction [3] and the hyperfine interaction with the lattice nuclei [4]. Recent experimental studies on different types of QDs have demonstrated long spin lifetimes $T_{1}$ on the order of a ms at cryogenic temperatures [5]. This has raised hopes that the $T_{2}$ times may be similarly long, with encouraging indications to that effect found lately [6]. 
Currently, the picture of spin physics in QDs is far from being complete. The electron spin can be characterized both by "static" and "dynamic" quantities. The "static" quantity is the $g$-factor, which is generally a tensor in a crystal. However, for the electron it is often assumed to be isotropic so that it could be treated as a scalar, as the conduction band states arise from $s$-like atomic orbitals. However, for semiconductors with band gaps in the $1-2 \mathrm{eV}$ range band mixing effects with, for example, the valence band formed by $p$-type atomic orbitals, are important. A well known consequence is the possibility of considerable deviation of the $g$-factor magnitude from its vacuum value of about 2 .

Dynamic quantities are the spin relaxation rates, which describe on what timescale a spin state that has been prepared at some time in a given state is destroyed. For a single spin the longitudinal and transverse spin relaxation times, $T_{1}$ and $T_{2}$, respectively, can be distinguished. $T_{1}$ describes the relaxation between the two spin split levels in a longitudinal magnetic field, while $T_{2}$ describes phase changes of the spin precession about a transverse magnetic field. Measurements of these times are ideally performed on a single spin under constant experimental conditions. This is often hard to fulfil experimentally.

Due to lack of sensitivity the experiments might have to be performed on multiple spins. For such an ensemble, in particular in a crystal environment, variations of the spin properties might occur, due to which a much faster decay of the spin phase is observed than indicated by the $T_{2}$ time. This ensemble spin phase decay is described by the dephasing time $T_{2}^{*}$. Another similar situation corresponds to long required measurement times during which statistical fluctuations of the environment occur, potentially leading also to experimental relaxation times shorter than the $T_{2}$.

\section{Experiment}

In this contribution we demonstrate that by pump-probe Faraday rotation spectroscopy [7] on singly charged QDs information about the aforementioned quantities can be obtained. Self-assembled (In,Ga)As/GaAs QD samples were subject of our experimental studies. Figure 1 shows an atomic force micrograph of a QD reference sample, which was not capped by GaAs, which is otherwise indispensable for a high optical quality. To obtain strong enough signal in the Faraday rotation, the samples contained 20 layers of QDs separated by $60 \mathrm{~nm}$ wide barriers. The layer dot density is about $10^{10} \mathrm{~cm}^{-2}$. For an average occupation by a single electron per dot, the structures were $n$-modulation doped $20 \mathrm{~nm}$ below each layer with a Si-dopant density about equal to the dot density. The sample was thermally annealed so that the QD ground state transition energy fits into the sensitivity range of Si photodetector. The ground state emission is located around $1.396 \mathrm{eV}$, as shown in the photoluminescence spectrum in Fig. 2, with a full width at half maximum of about $10 \mathrm{meV}$.

The sample was immersed in an optical split-coil magneto-cryostat at temperature $T=2 \mathrm{~K}$. For the Faraday rotation studies the magnetic field up to 


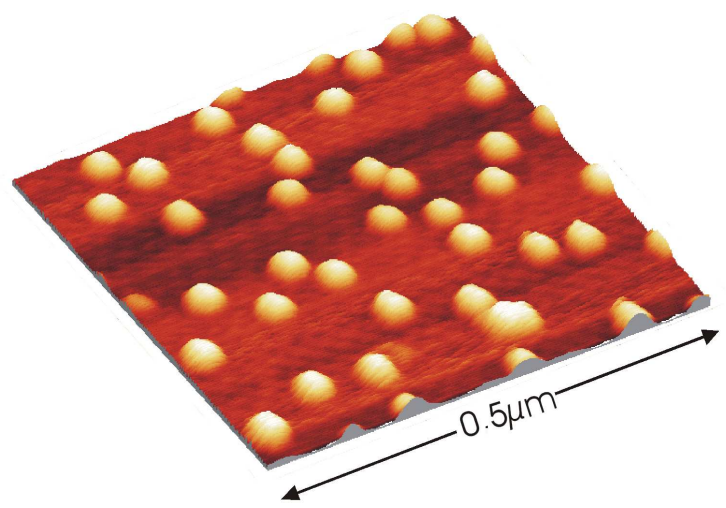

Fig. 1. Atomic force micrograph of a reference sample of uncapped self-assembled (In, Ga)As/GaAs QDs.

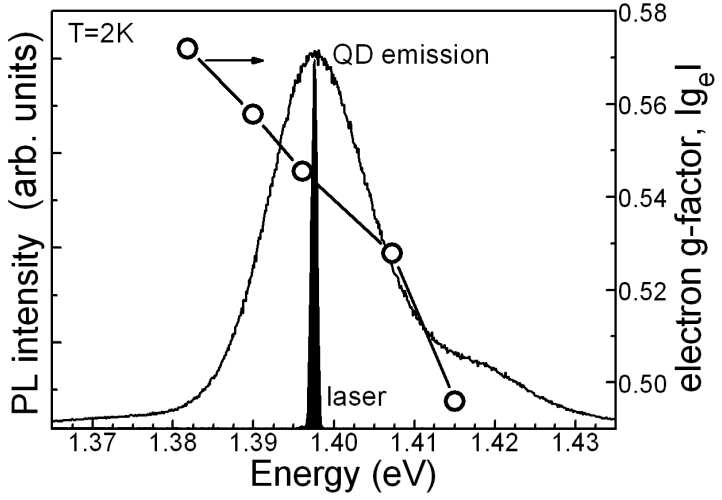

Fig. 2. Photoluminescence spectrum of the studied $n$-doped (In,Ga)As/GaAs QDs and spectrum of the exciting laser in the Faraday rotation pump-probe studies. The symbols give the electron $g$-factor (see right hand scale for values) as function of the luminescence emission energy.

$B=7 \mathrm{~T}$ was aligned perpendicular to the light propagation direction $z$ (Voigt configuration), which we take also as the quantization axis for the carrier angular momenta. A titanium-sapphire laser emitting pulses with duration of $\approx 1 \mathrm{ps}$ (full width at half maximum of $\approx 2 \mathrm{meV}$ ) at $75.6 \mathrm{MHz}$ repetition rate was used for optical excitation, hitting the sample along the growth direction. Therefore in case of left- (right-) circular polarization it can inject an electron with spin parallel (antiparallel) and a hole with momentum antiparallel (parallel) to the growth direction. Before illumination by the pump pulse, i.e. in equilibrium, the spin of the electron is oriented parallel or antiparallel to the magnetic field, so that no magnetization is present along the spin quantization axis. The laser was tuned to the QD ground state transition energy (see Fig. 1). The pulses were split into a circularly polarized pump component by which an imbalance in spin orientation along the 
growth axis is achieved and a linearly polarized probe component, by which this imbalance is tested. The imbalance leads to a difference of the refractive indices for left- and right-circularly light, which leads to a rotation of the linear polarization of the probe beam (circular birefringence). The rotation angle in our case is measured by a homodyne technique based on phase-sensitive balanced detection.

\section{Faraday rotation data}

Figure 3 shows the Faraday rotation signal of the $n$-doped QDs vs. delay between pump and probe for different magnetic fields, which were oriented in the QD plane along the assumed $x$-axis [8]. Quantum mechanically the precession corresponds to quantum beats between the two spin-split electron levels. Pronounced beats are observed with some additional modulation at high $B$, which is caused by the photoexcited carriers. These carriers disappear from the system though

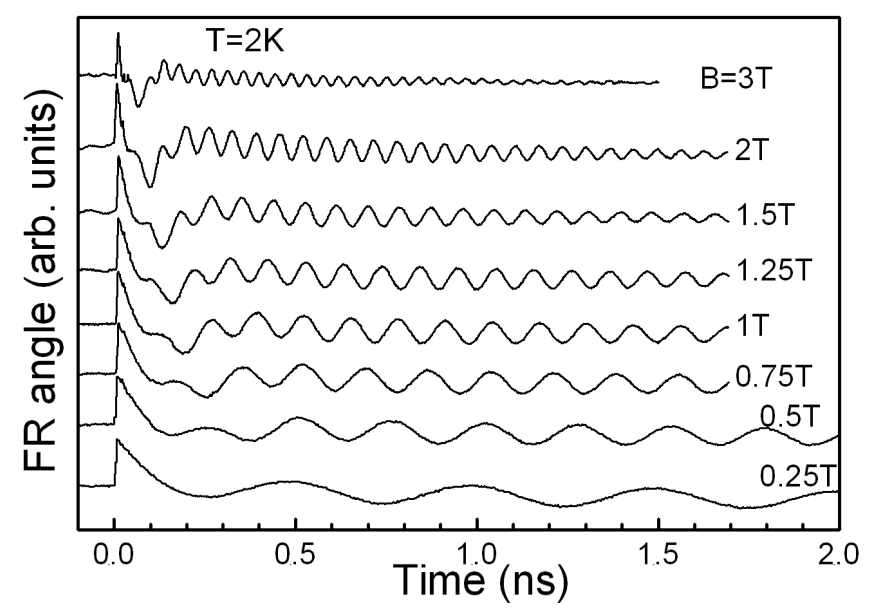

Fig. 3. Faraday rotation spectra as function of the delay between pump and probe for different magnetic field applied in the QD plane along the assumed $x$-direction.

radiative recombination. For this recombination we have determined a decay time of 400 ps through time-resolved photoluminescence. For a fixed magnetic field, the beats are damped with increasing delay time. This damping is much weaker at low fields than at high fields. For example, the oscillations last about 4 ns at $0.5 \mathrm{~T}$, confirming that the beats are due to the resident electrons. In contrast, at high fields the spin beats are fading away within a ns due to enhanced dephasing.

In order to observe these beats, obviously the initial spin orientation along the field must have been rotated through the pump pulse by 90 degrees to an orientation normal to the field. Any spin state along the field can be written as superposition of spin parallel and antiparallel to the light propagation direction. The reorientation of the electron spin occurs through excitation of an intermediate 
superposition state consisting of an electron whose spin orientation prevents absorption of the circularly light due to the Pauli blocking and the trion formed by light absorption. The trion decays at some point, leaving behind an electron with a well defined spin plus some contribution from the unpaired electron whose spin has precessed during the trion lifetime, which varies in the ensemble. By applying such a circularly polarized excitation, electron spin polarization can be accumulated. Calculations show that under optimum conditions a $99 \%$ polarization degree can be achieved by 12 excitation cycles only [8].

\section{Discussion}

Let us consider the Faraday rotation data in more detail. For that purpose, we have analyzed the Faraday rotation traces in Fig. 3 by an oscillatory function with exponentially damped amplitude, $\approx \exp \left(-t / T_{2}^{*}\right) \cos \left(\omega_{\mathrm{e}} t\right)$. As we address an ensemble, we assume that the decay is due to dephasing and therefore occurs on the time scale $T_{2}^{*}$. This will be substantiated further below. Two major points result from the analysis:

1) The beat oscillation frequency increases with magnetic field as expected from the spin splitting of electron states: $\hbar \omega_{\mathrm{e}}=g_{\mathrm{e}} \mu_{\mathrm{B}} B$, where $g_{\mathrm{e}}$ is the electron $g$-factor along the magnetic field direction and $\mu_{\mathrm{B}}$ is the Bohr magneton. The experimental magnetic field dependence of the electron precession frequency is

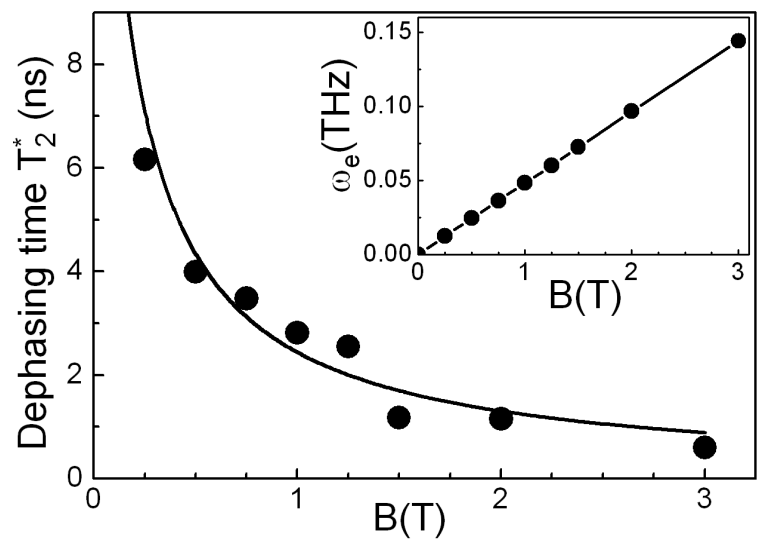

Fig. 4. Main panel: dephasing time $T_{2}^{*}$ as function of the magnetic field. The inset gives the field dependence of the electron precession frequency $\omega_{\mathrm{e}}$.

shown in the inset of Fig. 4. From a $B$-linear fit we obtain $\left|g_{\mathrm{e}}\right|=0.57$. Let us note that the sign of the $g$-factor cannot be determined from these data. The symbols in Fig. 2 show the variation of the electron $g$-factor when the laser pulse energy is varied across the inhomogeneously broadened emission band. From the low to the high energy side the $g$-factor modulus decreases from 0.57 to 0.49 . 
By varying the orientation of the magnetic field we are able to map out the directional dependence of the electron $g$-factor. For example, we find that the $g$-factor along the heterostructure growth direction is 0.61 which is substantially larger than the in-plane $g$-factor. This demonstrates already that the g-factor cannot be treated as scalar quantity.

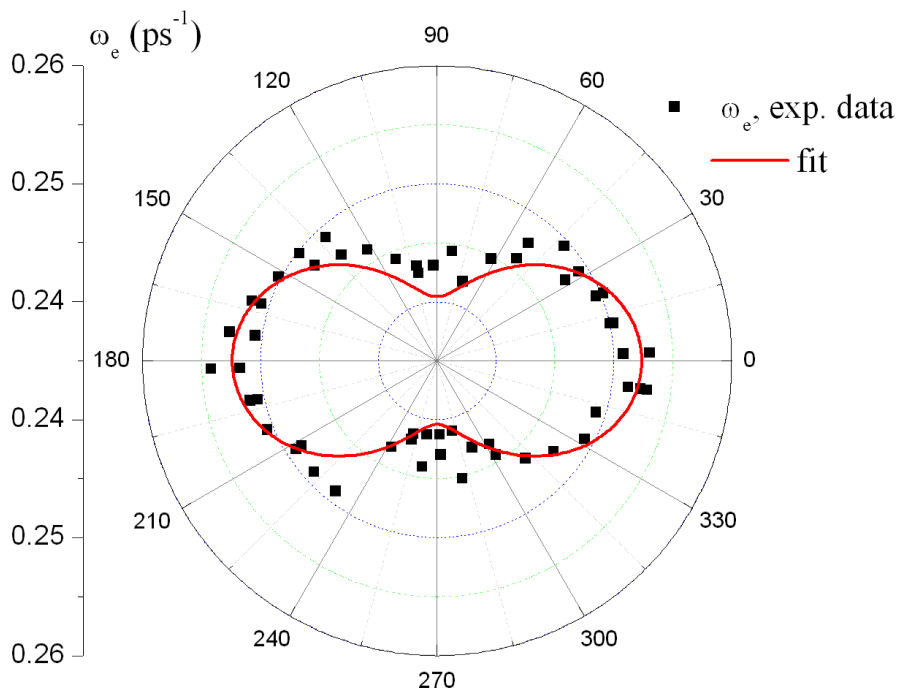

Fig. 5. In-plane (normal to the heterostructure growth direction) anisotropy of the electron spin precession frequency $\omega_{\mathrm{e}}$ in the (In,Ga)As/GaAs QDs.

This is further supported by the data in Fig. 5 which gives the variation of the electron precession frequency, when the magnetic field orientation is rotated in the QD plane. Also for this dependence we do not obtain a rotational symmetry, but rather a pattern with a twofold symmetry. When translating the frequency into the $g$-factor we find that it varies from 0.57 along the $x$-axis to 0.54 along the $y$-axis.

2) The decay time $T_{2}^{*}$ is plotted in Fig. 4 versus magnetic field. A considerable decrease occurs with increasing field which can be well described by a $1 / B$-dependence as the fit to the data shows (the line). The $T_{2}^{*}$ reflects the impact of the ensemble on the spin coherence: as described in the introduction, the coherence time $T_{2}$ should be ms, if it would reach its upper limit of $2 T_{1}$. However, in the ensemble much shorter times are observed, because all carriers start their precession with the same phase after initialization with the pump pulse, but variations $\Delta g_{\mathrm{e}}$ of the $g$-factor within the ensemble lead to a rather quick loss of phase coherence among the precessing electrons: an enhanced spread of the precession frequencies $\omega_{\mathrm{e}}$ with increasing $B$ occurs, as $\Delta \omega_{\mathrm{e}} \propto \Delta g_{\mathrm{e}} B$. Assuming that it is these variations which lead to the fact decay in magnetic field, their impact is described by $\left(T_{2}^{*}\right)^{-1}=\left(T_{2}\right)^{-1}+\Delta g_{\mathrm{e}} \mu_{\mathrm{B}} B / \hbar \sqrt{2}$, where the first term on the right 
hand side can be neglected as it is much smaller than the second contribution in non-zero field. The solid line in Fig. 4 shows a $1 / B$ fit to the experimental data, from which a $g$-factor variation of $\Delta g_{\mathrm{e}}=0.004$ is extracted for the QDs excited within the laser profile. From the data one can also conclude that $T_{2}^{*}$ is longer than 10 ns at $B=0$. The zero-field dephasing is mainly caused by spin precession about the frozen magnetic field of the dot nuclei [9]. The net orientation of all nuclei in a QD varies from dot to dot, and it is these variations that lead to ensemble dephasing at zero field.

In summary, we have demonstrated that pump-probe Faraday rotation spectroscopy allows one to obtain detailed insight into the properties of electron spins in quantum dots. As examples we have discussed the $g$-factor of the electron as well as the dephasing time within the QD ensemble.

\section{Acknowledgments}

This work was financially supported by the BMBF program "nanoquit", the DARPA program QuIST, the Office of Naval Research, and the Deutsche Forschungsgemeinschaft (Forschergruppe "Quantum Optics in Semiconductor Nanostructures"). R. Oulton thanks the Alexander von Humboldt foundation.

\section{References}

[1] D. Loss, D.P. diVincenzo, Phys. Rev. A 57, 120 (1998).

[2] A. Imamoglu, D.D. Awschalom, G. Burkard, D.P. diVincenzo, D. Loss, M. Sherwin, A. Small, Phys. Rev. Lett. 83, 4204 (1999).

[3] See, for example, T. Brandes, T. Vorrath, Phys. Rev. B 66, 075341 (2002).

[4] See, for example, A.V. Khaetskii, D. Loss, L. Glazman, Phys. Rev. Lett. 88, 186802 (2002).

[5] J.M. Elzerman, R. Hanson, L.H. Willems van Beveren, B. Witkamp, L.M. K. Vandersypen, L.P. Kouwenhoven, Nature 430, 431 (2004); M. Kroutvar, Y. Ducommun, D. Heiss, M. Bichler, D. Schuh, G. Abstreiter, J.J. Finley, Nature 432, 81 (2004).

[6] J.R. Petta, A.C. Johnson, J.M. Taylor, E.A. Laird, A. Yacoby, M.D. Lukin, C.M. Marcus, M.P. Hanson, A.C. Gossard, Science 309, 2180 (2005); A. Greilich, D.R. Yakovlev, A. Shabaev, Al.L. Efros, I.A. Yugova, R. Oulton, V. Stavarache, D. Reuter, A. Wieck, M. Bayer, Science 313, 341 (2006).

[7] Semiconductor Spintronics and Quantum Computation, Eds. D.D. Awschalom, D. Loss, N. Samarth, Springer-Verlag, Heidelberg 2002.

[8] A. Greilich, R. Oulton, E.A. Zhukov, I.A. Yugova, D.R. Yakovlev, M. Bayer, A. Shabaev, Al.L. Efros, I.A. Merkulov, V. Stavarache, D. Reuter, A. Wieck, Phys. Rev. Lett. 96, 227401 (2006).

[9] I. Merkulov, Al.L. Efros, M. Rosen, Phys. Rev. B 65, 205309 (2002). 